\title{
Adaptabilidade e estabilidade fenotípica de clones de cana-de-açúcar
}

\author{
William Rodrigues Antunes ${ }^{(1)}$, Edgar Ricardo Schöffel(1), Sergio Delmar dos Anjos e Silva ${ }^{(2)}$, \\ Eberson Eicholz ${ }^{(2)}$ e Adílson Härter ${ }^{(1)}$
}

\begin{abstract}
(1)Universidade Federal de Pelotas, Faculdade de Agronomia Eliseu Maciel, Campus Capão do Leão, CEP 96010-900 Capão do Leão, RS, Brasil. E-mail: wr_antunes@hotmail.com, ricardo.schoffel@gmail.com, adilsonharter@hotmail.com (2)Embrapa Clima Temperado, Rodovia BR-392, Km 78, 9 o Distrito, Monte Bonito, Caixa Postal 321, CEP 96010-971 Pelotas, RS, Brasil. E-mail: sergio.anjos@embrapa.br, eberson.eicholz@embrapa.br
\end{abstract}

Resumo - O objetivo deste trabalho foi determinar a adaptabilidade e a estabilidade de clones de cana-de-açúcar (Saccharum spp.) de ciclo médio-tardio, quanto à produção de colmos e de açúcar, e a eficiência da conversão de energia solar em produção, na região Noroeste do Estado do Rio Grande do Sul. Treze clones foram avaliados em nove ambientes, em delineamento de blocos ao acaso, com três repetições. As variáveis utilizadas para a análise de efeitos principais aditivos e interações multiplicativas (AMMI) foram: a produtividade de colmos e de açúcar, e a eficiência da conversão de energia solar em produção. Houve interação entre clones e ambientes, e o clone RB996527 destacou-se por ampla adaptação aos ambientes de teste, elevada produtividade de colmos e açúcar, estabilidade e eficiência da conversão de energia solar em produção, tendo superado a testemunha RB867515. Os ambientes com maior produtividade foram São Luiz Gonzaga (primeira-soca) e Santa Rosa (cana-planta e primeira-soca).

Termos para indexação: Saccharum, AMMI, ampla adaptação, interação clones x ambientes.

\section{Adaptability and phenotypic stability of sugarcane clones}

\begin{abstract}
The objective of this work was to assess the adaptability and stability of sugarcane (Saccharum spp.) clones of mid-late cycles, for their stem and sugar production, and their efficiency of solar energy conversion, in the northwestern region of the state of Rio Grande do Sul, Brazil. Thirteen clones were evaluated in nine environments, in a randomized complete block design, with three replicates. The variables used for the analysis of additive main effects and multiplicative interactions (AMMI) were: the stem and sugar productivity, and the efficiency of solar energy conversion. There was interaction between clones and environments, and the RB996527 clone stood out for its broad adaptation to test environments, high productivity of stalks and sugar, stability, and efficiency of solar energy conversion, having surpassed the control RB867515. The higher productivity environments were São Luiz Gonzaga (first ratoon) and Santa Rosa (plant cane and first ratoon).
\end{abstract}

Index terms: Saccharum, AMMI, broad adaptation, interaction clone x environment.

\section{Introdução}

A região Sul do Brasil, assim como o Centro-Oeste, é considerada uma das novas fronteiras agrícolas para o cultivo da cana-de-açúcar. Recentemente, o zoneamento agroecológico identificou cerca de 1,5 milhão de hectares com aptidão ao cultivo canavieiro, no Rio Grande do Sul (Manzatto et al., 2010). Assim, a região noroeste do Estado torna-se promissora, pois, nela situam-se as principais usinas de processamento de cana-de-açúcar, o que facilita o escoamento da produção. Atrelada à expansão da cultura, inclui-se a demanda por cultivares altamente responsivas, tanto em produção de colmos quanto em concentração de açúcar, para o cultivo em ambientes diversos (Silveira et al., 2012).
Para o desenvolvimento da cultura na região, é fundamental a disponibilidade de diferentes cultivares, bem como o conhecimento do comportamento ecofisiológico delas em cada ambiente de produção. O cultivo de cana-de-açúcar no Rio Grande do Sul é basicamente composto por clones e variedades muito antigas - multiplicadas muitas vezes como variedades crioulas -, que apresentam baixo rendimento e pouca tolerância a estresses abióticos (Rugeri, 2015). O estudo do desempenho de clones em ambientes de produção visa identificar as melhores combinações clone $\mathrm{x}$ ambiente, para obter a indicação mais precisa de um clone para uma determinada região, pois, para a cultura da cana-de-açúcar, no Rio Grande do Sul, há poucos estudos nesta linha de pesquisa (Verissimo et al., 2012). 
Uma peculiaridade da região Sul é a ocorrência de temperaturas baixas e risco de geadas no período que antecede a colheita de clones médio-tardios (Almeida et al., 2008). Isto ressalta a relevância de estudos que avaliem a adaptação desses genótipos a esse ambiente.

O estudo com clones de ciclo médio-tardio é de suma importância, pois, objetiva estender o período de industrialização, ao melhorar o desempenho da usina de processamento com oferta mais distribuída de matéria-prima ao longo da safra (Dinardo-Miranda et al., 2008).

O objetivo deste trabalho foi determinar a adaptabilidade e estabilidade de clones de cana-de-açúcar de ciclo médio-tardio, quanto à produção de colmos e de açúcar, e a eficiência da conversão de energia solar em produção, na região noroeste do Estado do Rio Grande do Sul.

\section{Material e Métodos}

A avaliação da produtividade de 13 clones de maturação médio-tardia - RB005003 (C1), RB005014 (C2), RB005017 (C3), RB006624 (C4), RB937570 (C5), RB965560 (C6), RB975290 (C7), RB995431 (C8), RB996519 (C9), RB996527 (C10), RB996532 (C11), RB867515 (C12-testemunha), RB92579 (C13) - foi realizada em cinco municípios da região noroeste do Rio Grande do Sul, cultivados como cana-planta e cana primeira-soca. Analisaram-se os dados de produtividade das safras 2012/2013 e 2013/2014, originados de experimentos de avaliação de clones de cana-de-açúcar, implantados pela Embrapa Clima Temperado, conduzidos pela Rede Interuniversitária para o Desenvolvimento do Setor Sucroenergético (Ridesa) e disponibilizados pelo Programa de Melhoramento Genético da Cana-de-açúcar (PMGCA) da Universidade Federal do Paraná, nos municípios de: Porto Xavier, $27^{\circ} 53^{\prime} \mathrm{S}, 55^{\circ} 10^{\prime} \mathrm{W}$, a $125 \mathrm{~m}$ de altitude; Salto do Jacuí, $29^{\circ} 00^{\prime} \mathrm{S}, 53^{\circ} 13^{\prime} \mathrm{W}$, a $361 \mathrm{~m}$ de altitude; São Luiz Gonzaga, $28^{\circ} 22^{\prime} \mathrm{S}, 54^{\circ} 54^{\prime} \mathrm{W}$, a $225 \mathrm{~m}$ de altitude; Santa Rosa, $27^{\circ} 50^{\prime} \mathrm{S}, 54^{\circ} 26^{\prime} \mathrm{W}$, a

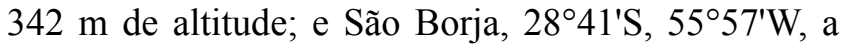
$97 \mathrm{~m}$ de altitude. Cada combinação município-corte foi considerada como um ambiente, com a seguinte nomenclatura: A1, cana-planta em Porto Xavier; A2, cana primeira-soca, em Porto Xavier; A3, cana-planta em Salto do Jacuí; A4, cana primeira-soca em Salto do Jacuí; A5, cana-planta em São Luiz Gonzaga; A6, cana primeira-soca em São Luiz Gonzaga; A7, cana-planta em Santa Rosa; A8, cana primeira-soca em Santa Rosa; e A9, cana primeira-soca em São Borja.

O preparo do solo foi realizado com uma aração e duas gradagens e, em seguida, com a formação dos sulcos, à profundidade de 30 a $40 \mathrm{~cm}$, e o espaçamento entre sulcos de 1,40 m. Para o plantio, utilizou-se o sistema convencional (manual), com a distribuição de toletes com três gemas ao longo dos sulcos, tendose colocado seis toletes por metro linear, no total de 18 gemas $\mathrm{m}^{-1}$, conforme o recomendado para cultura (Zambon \& Daros, 2005).

O plantio foi realizado na segunda quinzena de agosto de 2012, tendo-se feito adubação de base conforme a análise química do solo de cada local. Entre dezembro e janeiro, foi realizada a adubação de cobertura com $90 \mathrm{~kg} \mathrm{ha}^{-1}$ de N. No cultivo de cana primeira-soca, após a colheita dos experimentos, realizou-se a adubação de manutenção com $30 \mathrm{~kg} \mathrm{ha}^{-1}$ de $\mathrm{N}, 60 \mathrm{~kg} \mathrm{ha}^{-1}$ de $\mathrm{P}_{2} \mathrm{O}_{5}$ e $60 \mathrm{~kg} \mathrm{ha}^{-1}$ de $\mathrm{K}_{2} \mathrm{O}$.

Utilizou-se o delineamento experimental de blocos ao acaso, com três repetições, compostas por parcelas de quatro a seis linhas de $10 \mathrm{~m}$ de comprimento. A colheita da cana-planta foi feita entre julho e setembro de 2013, e da cana primeira-soca em julho de 2014, quando os clones apresentavam o índice de maturação entre 0,85 e 1,0 , que é o índice de cana madura (Cesnik \& Miocque, 2004).

As variáveis utilizadas para a análise AMMI (Zobel et al., 1988) foram: produtividade de colmos (TCH, $\mathrm{Mg} \mathrm{ha}^{-1}$ ); produtividade de açúcar (TSSTH, $\mathrm{Mg} \mathrm{ha}^{-1}$ ); e eficiência da conversão de energia solar em produção (Ef Rs, g MJ $^{-1}$ ). Para o cálculo da produtividade de colmos e açúcar, utilizaram-se fórmulas do manual de experimentação para a condução de experimentos (Zambon \& Daros, 2005), descritas a seguir:

Para o cálculo da $\mathrm{TCH}$, utilizou-se a fórmula $\mathrm{TCH}=(\mathrm{P} 10 \mathrm{C} / 10) \times(\mathrm{NCP} / 10) \times(10000) /(\mathrm{E}) /(1000)$, em que: P10C é a massa de 10 colmos $(\mathrm{kg})$; NCP é o número de colmos na parcela; e $\mathrm{E}$ é o espaçamento entre linhas.

A produtividade de açúcar foi obtida pela fórmula $\mathrm{TSSTH}=($ valor médio do SST $\times \mathrm{TCH}) / 100$, em que SST é a leitura média de sólidos solúveis totais obtida em três diferentes colmos, com a amostragem no terceiro entrenó abaixo do ponto de quebra do palmito e no terceiro entrenó acima do solo, com a utilização de um calador e leitura em refratômetro digital portátil. 
Para o cálculo da eficiência da conversão de energia solar em produção, adaptou-se a fórmula utilizada por Streck et al. (1998) - Ef Rs = (TCH/RAD)x(100) -, em que RAD é a radiação solar global acumulada da emergência à colheita.

Os dados meteorológicos de temperatura do ar, chuva e radiação solar global, utilizados no presente trabalho, foram disponibilizados pelo Instituto Nacional de Meteorologia (Inmet), $8^{\circ}$ Distrito de Meteorologia, com medições realizadas por estações automáticas localizadas em cada município.

Os dados fenométricos e de qualidade da cultura e os dados meteorológicos foram organizados em planilhas eletrônicas, e as análises de variância, análise AMMI e os gráficos biplot foram realizados por meio do programa estatístico SAS, versão 9.2 (SAS Institute, Cary, NC, EUA).

Utilizou-se, ainda, um referencial teórico adicional sobre clones e ambientes avaliados pelo método AMMI, denominado de clone e ambiente ideal (Ci e Ai), descrito por Pacheco et al. (2005), em que a superioridade agronômica de um clone deve ser avaliada comparativamente às produtividades máximas observadas em cada ambiente. Assim, um clone teoricamente ideal seria aquele que reunisse, ao longo dos ambientes de teste, a produtividade máxima.

\section{Resultados e Discussão}

$\mathrm{Na}$ análise conjunta dos experimentos, detectou-se a significância quanto à fonte de variação da interação entre clones e ambientes, para as três variáveis analisadas (Tabela 1).

A soma dos quadrados $\left(\mathrm{SQ}_{\mathrm{CXA}}\right)$ foi decomposta em oito eixos de CP(Componentes principais), e a variância acumulada no primeiro eixo IPCA("Interation principal component axes") explicou 36,3\% da TCH, 35,6\% da TSSTH e $39,0 \%$ da Ef Rs. Estes valores ficaram próximos aos encontrados por Verissimo et al. (2012) para TCH e TSSTH, porém, esses autores utilizaram os dois primeiros eixos de IPCA. Gauch (1988) relata que uma das premissas da análise AMMI é que no primeiro eixo há maior captação da percentagem de "padrão", ou seja, à medida que se eleva o número de eixos selecionados, aumenta-se a percentagem de "ruído", o que pode reduzir o poder de predição da análise AMMI. Assim, a interpretação gráfica foi realizada, tendo-se considerado apenas o biplot AMMI1 (IPCA1 $\times$ TCH); (IPCA1 × TSSTH); (IPCA1 × Ef Rs).

Os clones que se posicionaram mais próximos da origem, em relação ao eixo de interação entre clones e ambientes IPCA1, para a variável $\mathrm{TCH}$, foram: RB005003 (C1), RB005017 (C3), RB006624 (C4), RB996519 (C9), RB996527 (C10), RB996532 (C11), RB867515 (C12, testemunha). Este posicionamento indica alta estabilidade fenotípica de $\mathrm{TCH}$ e ampla adaptação aos ambientes de teste (Figura 1 A). Destacaram-se os clones C9 e C10, que superaram a média geral de TCH $\left(120,7 \mathrm{Mg} \mathrm{ha}^{-1}\right)$, e alcançaram, respectivamente, a terceira $\left(127,9 \mathrm{Mg} \mathrm{ha}^{-1}\right)$ e a quinta $\left(125,3 \mathrm{Mg} \mathrm{ha}^{-1}\right)$ posições gerais. No Estado de São Paulo, Fernandes Junior et al. (2013) também verificaram alta estabilidade em TCH para a testemunha RB867515, corroborada pelos resultados encontrados no presente trabalho.

Em geral, os clones - C6, C5, C9, C13, C10, C2, C7, $\mathrm{C} 4, \mathrm{C} 3$ e C1 - apresentaram médias de produtividade de colmos maiores do que a testemunha $(\mathrm{C} 12)$ em 13,18, 13,01, 10,93, 9,45, 8,67, 7,63, 4,34, 3,30, 1,56 e 1,56\%, respectivamente (Tabela 2), e os clones C9, C10, C4, $\mathrm{C} 3$ e C1 foram estáveis e produtivos. Fernandes Junior et al. (2013) identificaram clones com produtividade de colmos maior do que as testemunhas utilizadas, em 3,5 e $7 \%$, respectivamente, e apenas três clones apresentaram estabilidade produtiva.

Os clones que mais contribuíram para interação entre clone e ambiente (baixa estabilidade), em TCH, foram: RB005014 (C2), RB937570 (C5), RB92579 (C13) e RB975290 (C7), pois apresentaram a maior magnitude de escore no eixo de interação IPCA1, o que indica variação de produtividade de colmos destes clones entre os ambientes. Entre estes, destacou-se o clone $\mathrm{C} 5$ com a segunda posição geral quanto à $\mathrm{TCH}$, com $130,3 \mathrm{Mg} \mathrm{ha}^{-1}$, valor muito próximo dos $130,5 \mathrm{Mg} \mathrm{ha}^{-1}$ produzidos pelo primeiro colocado $\mathrm{C} 6$.

Observação semelhante foi relatada por Fernandes Junior et al. (2013), em São Paulo, que verificaram elevadas médias de TCH e baixa estabilidade para alguns dos clones estudados.

Quanto à produtividade de açúcar (TSSTH) (Figura $1 \mathrm{~B}$ ), verifica-se que os clones mais estáveis e adaptados aos ambientes de teste foram: RB005003 (C1), RB005017 (C3), RB006624 (C4), RB965560 (C6), RB996527 (C10) e RB92579 (C13), os quais se posicionaram mais próximos da origem, em 
relação ao eixo de interação clone e ambiente IPCA1. Este resultado confirma a estabilidade e adaptação observada em $\mathrm{TCH}$, para os clones $\mathrm{C} 1, \mathrm{C} 3, \mathrm{C} 4$ e C10, o que indica alta produtividade tanto de colmos como de açúcar. Destacaram-se os clones C6, C10, C1 e C13, com médias de TSSTH superiores à média geral $\left(22,3 \mathrm{Mg} \mathrm{ha}^{-1}\right)$, que alcançaram a primeira $\left(24 \mathrm{Mg} \mathrm{ha}^{-1}\right)$, primeira (24 $\left.\mathrm{Mg} \mathrm{ha}^{-1}\right)$, quinta $\left(22,9 \mathrm{Mg} \mathrm{ha}^{-1}\right)$ e sexta $\left(22,5 \mathrm{Mg} \mathrm{ha}^{-1}\right)$ posições gerais, respectivamente, o que evidencia o elevado acúmulo de sólidos solúveis totais dos clones $\mathrm{C} 6$ e $\mathrm{C} 10$.

De maneira geral, os clones (C10, C6, C9, C5, C1, C2, C13, C7 e C4) apresentaram médias de produtividade de açúcar maiores que a testemunha (C12) na ordem de $13,39,13,39,12,91,12,44,8,19,6,30,6,30,3,94$ e 3,46\%, respectivamente (Tabela 2). Entretanto, os clones C10, C6, C1, C13 e C4 foram estáveis e os mais produtivos. Silva (2008), no Estado de São Paulo, verificou resultado semelhante identificando clones de ciclo médio-tardio com produtividade de açúcar acima das testemunhas, na ordem de 18 e 9,4\%, respectivamente, mas apenas um clone foi estável.

Os clones que mais contribuíram para a interação entre clone e ambiente (baixa estabilidade) foram: RB005014 (C2), RB937570 (C5) e RB975290(C7), pois apresentaram a maior magnitude de escore no eixo de interação IPCA1, indicando variação destes na produção de açúcar entre os ambientes. Entre eles destacaram-se com elevada média de TSSTH os clones $\mathrm{C} 2$ e C5, que atingiram, respectivamente, a sexta $\left(22,9 \mathrm{Mg} \mathrm{ha}^{-1}\right)$ e a quarta $\left(23,8 \mathrm{Mg} \mathrm{ha}^{-1}\right)$ posições gerais.

Quanto ao uso de energia solar em produção (Ef Rs) (Figura $1 \mathrm{C}$ ), observou-se que os clones mais estáveis e adaptados aos ambientes de teste foram: RB005003
(C1), RB005017 (C3), RB006624 (C4), RB996519 (C9), RB996527 (C10), RB996532 (C11) e RB867515 (C12, testemunha), que se posicionaram mais próximos da origem do eixo IPCA1, o que confirma o desempenho obtido por esses clones quanto ao $\mathrm{TCH}$ e é indicativo da elevada eficiência para converter energia solar em produção de colmos. Com médias de Ef Rs superiores à média geral $\left(2,13 \mathrm{~g} \mathrm{MJ}^{-1}\right)$, destacaram-se os genótipos C9 e C10 que atingiram, respectivamente, a terceira $\left(2,27 \mathrm{~g} \mathrm{MJ}^{-1}\right)$ e a quarta $\left(2,22 \mathrm{~g} \mathrm{MJ}^{-1}\right)$ posições gerais,.

Os clones C6, C5, C9, C13, C10, C2, C7, C4, C3 e $\mathrm{C} 1$ foram mais eficientes na conversão de energia solar em produção do que a testemunha (C12), em 13,79, 13,30, 11,82, 9,36, 9,36, 7,88, 4,43, 3,94, 1,97 e 1,97\%, respectivamente (Tabela 2). No entanto, os clones C9, $\mathrm{C} 10, \mathrm{C} 4, \mathrm{C} 3$ e $\mathrm{C} 1$ foram estáveis e eficientes, o que confirma o resultado obtido para produtividade de colmos.

Os clones que mais contribuíram para a interação clone $\mathrm{x}$ ambiente (baixa estabilidade) foram: RB005014 (C2), RB937570 (C5), e RB92579 (C13), pois apresentaram a maior magnitude de escore no eixo IPCA1, o que evidencia a grande variação destes clones entre os ambientes. Entre eles, destacou-se o clone RB937570 (C5) com elevada média de Ef Rs, ao alcançar a segunda $\left(2,30 \mathrm{~g} \mathrm{MJ}^{-1}\right)$ posição geral, com grande eficiência na conversão de energia solar em produção.

O clone RB965560 (C6) foi o que mais se aproximou do clone ideal $(\mathrm{Ci})$, quanto à produtividade de colmos e de açúcar e eficiência da conversão de energia solar em produção, em razão de sua elevada média de $\mathrm{TCH}$, TSSTH e Ef Rs, tendo alcançado a primeira posição geral nas três variáveis. Entretanto, considerando-se os critérios de estabilidade, produtividade e eficiência

Tabela 1. Análise de variância conjunta, para a produtividade de colmos (TCH), produtividade de açúcar (TSSTH) e eficiência da conversão de energia solar em produção (Ef Rs) de 13 clones de cana-de-açúcar, de maturação médio-tardia.

\begin{tabular}{|c|c|c|c|c|c|c|c|c|c|c|c|c|}
\hline \multirow[t]{2}{*}{ Fonte de variação } & \multicolumn{4}{|c|}{$\mathrm{TCH}$} & \multicolumn{4}{|c|}{ TSSTH } & \multicolumn{4}{|c|}{ Ef Rs } \\
\hline & GL & $\mathrm{QM}$ & $\mathrm{F}$ & $\operatorname{Pr}>F$ & GL & QM & $\mathrm{F}$ & $\operatorname{Pr}>\mathrm{F}$ & $\mathrm{GL}$ & QM & $\mathrm{F}$ & $\operatorname{Pr}>F$ \\
\hline Blocos/ambiente & 16 & 155,4 & 1,7 & 0,0521 & 16 & 4,4 & 1,1 & 0,3212 & 16 & 0,05 & 1,7 & 0,0362 \\
\hline Clones (C) & 12 & $1.532,2$ & 16,6 & $0,0001 * *$ & 12 & 50,2 & 13,0 & $0,0001 * *$ & 12 & 0,47 & 16 & $0,0001 * *$ \\
\hline Ambiente (A) & 8 & $30.251,7$ & 327 & $0,0001 * *$ & 8 & 682,3 & 176,6 & $0,0001 * *$ & 8 & 10,99 & 378 & $0,0001 * *$ \\
\hline Interação CxA & 96 & 868,6 & 9,39 & $0,0001 * *$ & 96 & 27,4 & 7,1 & $0,0001 * *$ & 96 & 0,28 & 9,51 & $0,0001 * *$ \\
\hline Resíduo & 232 & 96,8 & - & - & 232 & 3,9 & - & - & 232 & 0,03 & - & - \\
\hline IPCA 1 (padrão) & 19 & 531,3 & 16,5 & $0,0000 * *$ & 19 & 16,5 & 12,6 & $0,0000 * *$ & 19 & 0,18 & 17,8 & $0,0000 * *$ \\
\hline Resíduo AMMI1 & 77 & 229,9 & 7,1 & $0,0000^{* *}$ & 77 & 7,3 & 5,6 & $0,0000^{* *}$ & 77 & 0,07 & 6,9 & $0,0000 * *$ \\
\hline Erro médio/r & 232 & 32,3 & - & - & 232 & 1,3 & - & - & 232 & 0,01 & - & - \\
\hline
\end{tabular}

**Significativo a $1 \%$ de probabilidade, pelo teste F. Clones avaliados em nove ambientes, na região Noroeste do Estado do Rio Grande do Sul, com desdobramento da interação original CxA, pelo modelo AMMI. 

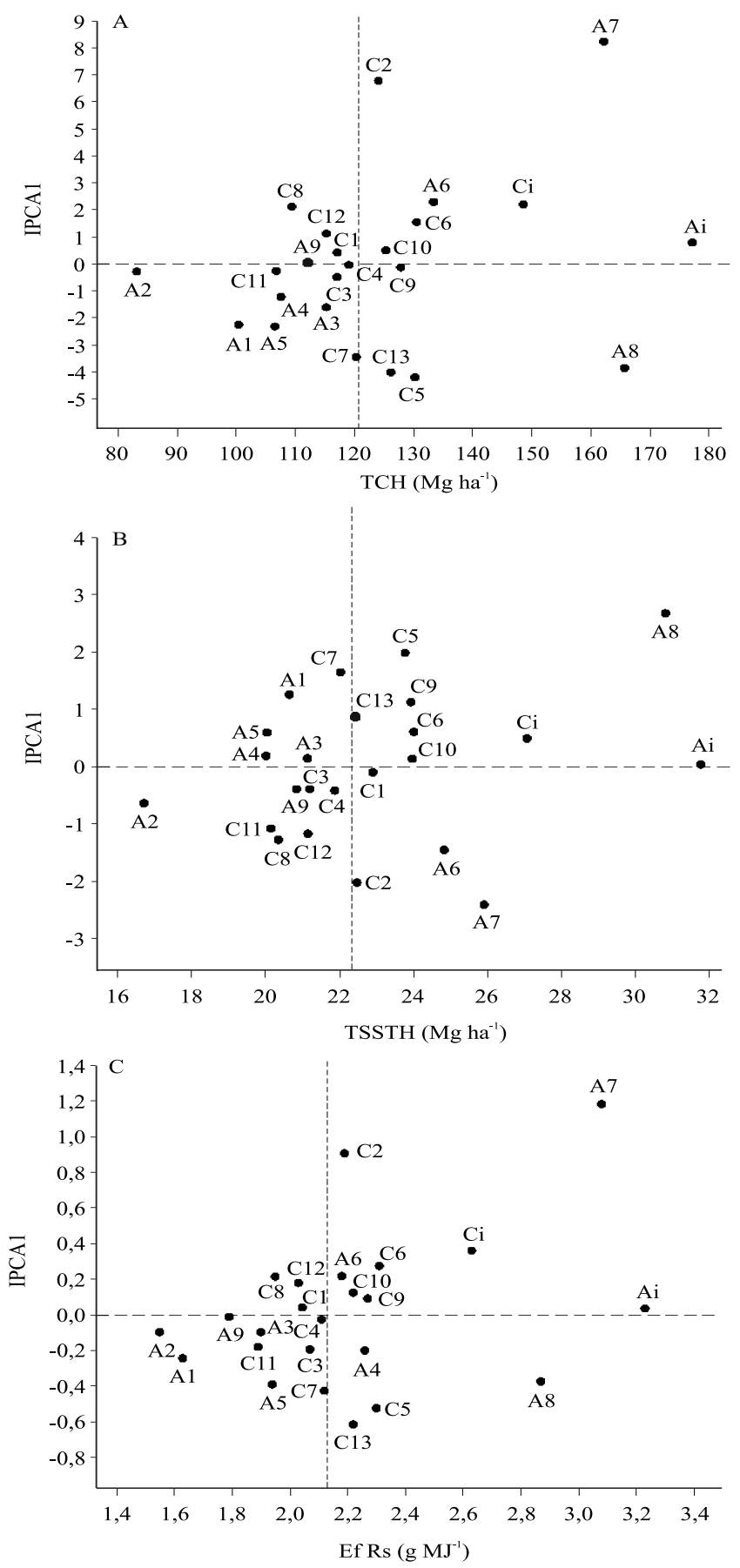

Figura 1. Biplot AMMI1 para: A, produtividade de colmos (TCH); B, produtividade de açúcar (TSSTH); e C, eficiência da conversão de energia solar em produção (Ef Rs) de 13 clones de cana-de-açúcar, de maturação médio-tardia, avaliados em nove ambientes, na região Noroeste do Estado do Rio Grande do Sul. A1-A9, local e estádio (cana-planta ou cana-soca); C1-C13, identificação do clone; Ai, ambiente ideal; e Ci, clone ideal. de conversão, o melhor desempenho foi o do clone RB996527 (C10).

Os ambientes de maior destaque quanto à produtividade de colmos e açúcar e eficiência da conversão de energia solar em produção, por médias superiores à média geral, foram: A6, São Luiz Gonzaga, primeira-soca; A7, Santa Rosa, cana-planta; e A8, Santa Rosa, primeira-soca. O ambiente A4 (Salto do Jacuí, primeira-soca) apresentou média superior à geral quanto à conversão da energia solar em produção. Os ambientes A3 (Salto do Jacuí, cana-planta), A4 (Salto do Jacuí, primeira-soca) e A9 (São Borja, primeira-soca) destacaram-se quanto à estabilidade, pois se aproximam do eixo de interação IPCA1, entretanto, sua produtividade de colmos e açúcar e a eficiência da conversão de energia solar em produção foram inferiores à média geral, com exceção do A4, conforme já citado.

Considerando-se a média das duas safras (2012/2013 e 2013/2014), o ambiente que apresentou a menor quantidade de radiação solar global acumulada foi Porto Xavier $\left(5.058,1 \mathrm{MJ} \mathrm{m}^{-2}\right)$, o que comprova a menor produtividade de colmos deste ambiente, enquanto a maior quantidade de radiação solar foi observada em São Borja (6.256,2 $\left.\mathrm{MJ} \mathrm{m}^{-2}\right)$, porém, em razão do menor volume de chuvas $(1.107,2 \mathrm{~mm})$, este ambiente não esteve entre os mais produtivos, pois apresentou TCH de 112,1 $\mathrm{Mg} \mathrm{ha}^{-1}$ e de TSSTH de 20,8 $\mathrm{Mg} \mathrm{ha}^{-1}$. Na média das duas safras, São Luiz Gonzaga apresentou o maior volume de chuvas $(1.925,7 \mathrm{~mm})$, fato que associado a uma boa disponibilidade de radiação solar global $\left(5.804,0 \mathrm{MJ} \mathrm{m}^{-2}\right)$ conferiu a este ambiente uma das melhores produtividades, com TCH de $120,0 \mathrm{Mg} \mathrm{ha}^{-1}$ e de TSSTH de 22,3 $\mathrm{Mg} \mathrm{ha}^{-1}$.

Os ambientes que mais contribuíram para a interação entre clone e ambiente (baixa estabilidade) foram A7 e A8, pois apresentaram a maior magnitude de escore no eixo de interação IPCA1, o que é indicativo de uma grande variação produtiva entre os clones nesses ambientes.

Em comparação com o ambiente ideal (Ai), o ambiente que mais se aproximou em produtividade de colmos (TCH) e produtividade de açúcar (TSSTH) foi A8 (Santa Rosa, primeira-soca) e, quanto à eficiência da conversão de energia solar em produção (Ef Rs), foi o A7 (Santa Rosa, cana-planta). Quanto à estabilidade, produtividade e eficiência de conversão, o mais próximo do ambiente ideal (Ai) foi o A3 (Salto do Jacuí, cana-planta). 
Tabela 2. Valores médios e ganho em relação à testemunha, para tonelada de colmos por hectare (TCH), tonelada de sólidos solúveis totais por hectare (TSSTH) e eficiência da conversão de energia solar em produção (Ef Rs) de 12 clones médio-tardios, em nove ambientes da região Noroeste do Estado do Rio Grande do Sul.

\begin{tabular}{|c|c|c|c|c|c|c|c|}
\hline Clone & ID & $\mathrm{TCH}$ & $\mathrm{Ganho}^{(1)}(\%)$ & TSSTH & Ganho $^{(1)}(\%)$ & Ef Rs & Ganho $^{(1)}(\%)$ \\
\hline RB005003 & $\mathrm{C} 1$ & 117,1 & 1,56 & 22,9 & 8,19 & 2,07 & 1,97 \\
\hline RB005014 & $\mathrm{C} 2$ & 124,1 & 7,63 & 22,5 & 6,30 & 2,19 & 7,88 \\
\hline RB005017 & $\mathrm{C} 3$ & 117,1 & 1,56 & 21,2 & 0,16 & 2,07 & 1,97 \\
\hline RB006624 & $\mathrm{C} 4$ & 119,1 & 3,30 & 21,9 & 3,46 & 2,11 & 3,94 \\
\hline RB937570 & $\mathrm{C} 5$ & 130,3 & 13,01 & 23,8 & 12,44 & 2,30 & 13,30 \\
\hline RB965560 & C6 & 130,5 & 13,18 & 24,0 & 13,39 & 2,31 & 13,79 \\
\hline RB975290 & $\mathrm{C} 7$ & 120,3 & 4,34 & 22,0 & 3,94 & 2,12 & 4,43 \\
\hline RB995431 & $\mathrm{C} 8$ & 109,4 & $-5,12$ & 20,4 & $-3,62$ & 1,94 & $-4,43$ \\
\hline RB996519 & $\mathrm{C} 9$ & 127,9 & 10,93 & 23,9 & 12,91 & 2,27 & 11,82 \\
\hline RB996527 & $\mathrm{C} 10$ & 125,3 & 8,67 & 24,0 & 13,39 & 2,22 & 9,36 \\
\hline RB996532 & $\mathrm{C} 11$ & 106,8 & $-7,37$ & 20,1 & $-5,04$ & 1,89 & $-6,90$ \\
\hline RB867515, testemunha & $\mathrm{C} 12$ & 115,3 & 0,00 & 21,2 & 0,00 & 2,03 & 0,00 \\
\hline RB92579 & $\mathrm{C} 13$ & 126,2 & 9,45 & 22,5 & 6,30 & 2,22 & 9,36 \\
\hline
\end{tabular}

${ }^{(1)}$ Ganho percentual de TCH, TSSTH e Ef Rs em relação à média da testemunha.

\section{Conclusões}

1. Os clones de cana-de-açúcar (Saccharum spp.) avaliados apresentam interação com os ambientes de cultivo.

2. O clone RB996527 (G10) apresenta elevada produtividade de colmos e açúcar, eficiência da conversão de energia solar em produção, estabilidade e ampla adaptação aos ambientes da região noroeste do Rio Grande do Sul.

3. Santa Rosa, RS, é o ambiente mais produtivo tanto em ciclo de cana-planta quanto em cana primeira-soca.

\section{Agradecimentos}

À Financiadora de Estudos e Projetos (Finep, processo 21400.09/0088-8), à Petróleo Brasileiro S.A (Petrobras, processo 100200.13/0180-2) e à Coordenação de Aperfeiçoamento de Pessoal de Nível Superior (Capes), pelo apoio financeiro.

\section{Referências}

ALMEIDA, A.C. dos S.; SOUZA, J.L.; TEODORO, I.; BARBOSA, G.V.S.; MOURA FILHO, G.; FERREIRA JÚNIOR, R.A. Desenvolvimento vegetativo e produção de variedades de cana-de-açúcar em relação à disponibilidade hídrica e unidades térmicas. Ciência e Agrotecnologia, v.32, p.1441-1448, 2008. DOI: $10.1590 / \mathrm{S} 1413-70542008000500013$.
CESNIK, R.; MIOCQUE, J. Melhoramento da cana-de-açúcar. Brasília: Embrapa Informação Tecnológica; Jaguariúna: Embrapa Meio Ambiente, 2004. 307p.

DINARDO-MIRANDA, L.L.; VASCONCELOS, A.C. . de; LANDELL, M. G. de A. (Ed.). Cana-de-açúcar. Campinas: Instituto Agronômico, 2008. v.1, 882p.

FERNANDES JUNIOR, A.R.; ANDRADE, J.A. da C.; SANTOS, P.C. dos; HOFFMANN, H.P; CHAPOLA, R.G; CARNEIRO, M.S; CURSI, D.E. Adaptabilidade e estabilidade de clones de cana-de-açúcar. Bragantia, v.72, p.208-216, 2013. DOI: 10.1590/ brag.2013.033.

GAUCH JR., H.G. Model selection and validation for yield trials with interaction. Biometrics, v.44, p.705-715, 1988.

MANZATTO, C.V.; BACA, J.F.M.; PEREIRA, S.E.M.; ASSAD, E.D.; MEIRELLES, M.S.P.; BACA, A.F.M.; NAIME, U.J.; MOTTA, P.A.F. da. Zoneamento agroecológico da cana-de-açúcar: abordagem metodológica para integração temática de grandes áreas territoriais. In: PRADO, R.B.; TURETTA, A.P.D.; ANDRADE, A.G. de. (Org.). Manejo e conservação do solo e da água no contexto das mudanças ambientais. Rio de Janeiro: Embrapa Solos, 2010. p.193-214.

PACHECO, R.M.; DUARTE, J.B.; VENCOVSKY, R.; PINHEIRO, J.B.; OLIVEIRA, A.B. Use of supplementary genotypes in AMMI analysis. Theoretical and Applied Genetics, v.110, p.812-818, 2005. DOI: 10.1007/s00122-004-1822-6.

RUGERI, A. P. Identificação do uso e desempenho de genótipos de cana-de-açúcar no estado do Rio Grande do Sul. 2015. 90p. Dissertação (Mestrado) - Universidade Federal de Pelotas, Pelotas.

SILVA, M. de A. Interação genótipo $\mathrm{x}$ ambiente $\mathrm{e}$ estabilidade fenotípica de cana-de-açúcar em ciclo de cana 
de ano. Bragantia, v.67, p.109-117, 2008. DOI: 10.1590/ S0006-87052008000100013.

SILVEIRA, L.C.I. da; KIST, V.; PAULA, T.O.M. de; BARBOSA, M.H.P.; OLIVEIRA, R.A. de; DAROS, E. Adaptabilidade e estabilidade fenotípica de genótipos de cana-de-açúcar no estado de Minas Gerais. Ciência Rural, v.42, p.587-593, 2012. DOI: 10.1590/S0103-84782012000400002.

STRECK, N.A.; BURIOL, G.A.; ADRIOLO, J.L.; SANDRI, M.A. Influência da densidade de plantas e da poda apical drástica na produtividade do tomateiro em estufa de plástico. Pesquisa Agropecuária Brasileira, v.33, p.1105-1112, 1998.
VERISSIMO, M.A.A.; SILVA, S.D. dos A. e; AIRES, R.F.; DAROS, E.; PANZIERA, W. Adaptabilidade e estabilidade de genótipos precoces de cana-de-açúcar no Rio Grande do Sul. Pesquisa Agropecuária Brasileira, v.47, p.561-568, 2012. DOI: 10.1590/S0100-204X2012000400012.

ZAMBON, J.L.C.; DAROS, E. Manual de experimentação para a condução de experimentos. Curitiba: UFPR, 2005. 49p.

ZOBEL, R.W.; WRIGHT, M.J.; GAUCH, H.G. Statistical analysis of a yield trial. Agronomy Journal, v.80, p.388-393, 1988. DOI: 10.2134/agronj1988.00021962008000030002x.

Recebido em 16 de junho de 2015 e aprovado em 18 de janeiro de 2016 